\title{
Papel protector de las lipoproteínas de alta densidad en sepsis: aspectos básicos e implicancias clínicas
}

\author{
Susana Contreras-Duarte, Pablo Varas, Fernanda Awad, Dolores Busso y Attilio Rigotti
}

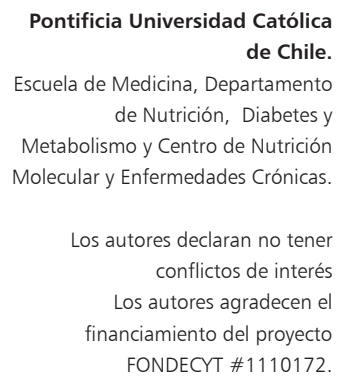

Pontificia Universidad Católica de Chile.

Escuela de Medicina, Departamento de Nutrición, Diabetes y Metabolismo y Centro de Nutrición Molecular y Enfermedades Crónicas.

Los autores declaran no tener conflictos de interés

Los autores agradecen el financiamiento del proyecto FONDECYT \# 1110172.

Recibido: 6 de marzo de 2013 Aceptado: 21 de noviembre de

Correspondencia a: Attilio Rigotti Rivera arigotti@med.puc.cl.

\author{
Protective role of high density lipoproteins in sepsis: \\ basic issues and clinical implications
}

High density lipoproteins (HDL) are responsible of reverse cholesterol transport and play an important antiatherogenic role. In recent years, several studies suggest that HDL have additional functions, including a possible anti-inflammatory activity in infectious conditions. Furthermore, available evidence indicates that the presence of lipopolysaccharide (LPS) within the circulation during infectious states induced by gram-negative bacteria may be involved in the decrease in HDL cholesterol levels and changes in lipoprotein composition, which have been associated with a higher mortality due to sepsis in animal models and in humans. In this article, we review this subject and also discuss possible mechanisms that explain the positive impact achieved by native HDL, reconstituted HDL, or HDL apolipoprotein peptides on the inflammatory response and mortality in models of endotoxemia. In this regard, it has been proposed that one of the mechanisms by which HDL protect against sepsis may be mediated by its binding ability and/or neutralizing capacity on LPS, avoiding an excessive response of the immune system. Thus, increasing blood levels of HDL and/or parenteral HDL administration may represent a new anti-inflammatory tool for managing septic states in humans.

Key words: High density lipoproteins (HDL), reconstituted HDL (rHDL), apo A-I mimetic peptide (L-4F), apolipoprotein A-I (apo A-I), lipopolysaccharide (LPS), interleukin-6 (IL-6), tumor necrosis factor $\alpha$ (TNF- $\alpha$ ).

Palabras clave: Lipoproteínas de alta densidad (HDL), HDL reconstituida (rHDL), péptido mimético de apo A-I (L-4F), apolipoproteína A-I (apo A-I), lipopolisacárido (LPS), interleuquina-6 (IL-6), factor de necrosis tumoral $\alpha($ FNT- $\alpha)$.

L as lipoproteínas son complejos macromoleculares compuestos por proteínas y lípidos que transportan compuestos hidrofóbicos en el medio acuoso de la circulación sanguínea de un organismo. Habitualmente, estos complejos son esféricos, hidrosolubles y están formados por un núcleo de lípidos apolares (colesterol esterificado y triglicéridos) cubierto con una capa externa anfipática constituida por apolipoproteínas, fosfolípidos y colesterol libre. En el plasma humano existen cuatro clases predominantes de lipoproteínas clasificadas, de acuerdo a sus densidades, en: quilomicrones, VLDL (very low density lipoproteins), LDL (low density lipoproteins) y HDL (high density lipoproteins). Las distintas clases de lipoproteínas se diferencian en su tamaño, composición lipídica y proteica, movilidad electroforética y funcionalidad. En el torrente sanguíneo, las diferentes clases de lipoproteínas transportan los lípidos desde el lugar donde son absorbidos o sintetizados hacia los distintos tipos celulares, donde son reconocidas por receptores específicos para apolipoproteínas presentes en la superficie celular.

\section{Composición, metabolismo y funcionalidad de HDL}

Las HDL se forman como partículas nacientes con la secreción intestinal y hepática de la apolipoproteína A-I (apo A-I), o bien mediante su liberación desde lipoproteínas ricas en triglicéridos. Posteriormente, la apo A-I capta fosfolípidos desde las células pasando a formar partículas de HDL discoidales conocidas como pre $\beta$-HDL. Estas partículas de HDL interactúan con el transportador ABCA1 (ATP-binding cassette transporter class A, type 1) de la superficie de las células captando más fosfolípidos y colesterol no esterificado. En seguida, la enzima LCAT (lecithin-cholesterol acyltransferase) esterifica el colesterol y lo moviliza hacia el centro de las partículas de HDL, las cuales adquieren mayor tamaño y forma esférica, denominándoseles $\alpha$-HDL. Las HDL culminan su maduración captando más colesterol no esterificado de origen celular por acción del receptor de HDL SR-BI (scavenger receptor class $B$, type I) y el transportador ABCG1 (ATP-binding cassette sub-family $G$ member 1). Las HDL maduras se 
clasifican en HDL2 y HDL3, siendo éstas últimas de mayor densidad, menor tamaño y menor contenido de colesterol.

El colesterol presente en las células de los tejidos periféricos es transportado mediante las partículas de HDL hasta el hígado por el proceso conocido como transporte reverso de colesterol. Este flujo reverso de colesterol puede ocurrir en forma directa mediante la interacción entre las partículas de HDL con el receptor SR-BI en el hígado o en forma indirecta a través de su traspaso hacia las lipoproteínas no HDL mediado por acción de la enzima de transferencia de ésteres de colesterol CETP (Cholesterylester transfer protein). El colesterol transportado en forma reversa hacia el hígado es excretado del organismo como tal o transformado en sales biliares, a través de la vía biliar.

Además de las enzimas remodeladoras ya mencionadas, las HDL están compuestas por otra variedad de enzimas que le permiten llevar a cabo cada una de las funciones en las que están involucradas estas partículas. Las HDL transportan una gran batería de enzimas encargadas principalmente de mantener el equilibrio oxidativo y disminuir la inflamación; entre ellas se encuentra la paraoxonasa 1 PON1 (paraoxonase 1), el factor activador de plaquetas acetil hidrolasa PAF-AH (Platelet-activating factor acetylhydrolase), la ceruloplasmina y la transferrina. Otros componentes de las HDL que se han descrito que participan en la prevención de la muerte celular son la apo A-I y la esfingosina-1-fosfato (S1P).

El transporte reverso de colesterol -definido como el flujo de colesterol desde los tejidos periféricos hacia el hígado- llevado a cabo por las partículas de HDL permite mantener la homeostasis de colesterol corporal y previene el desarrollo de ateroesclerosis, ya sea evitando que un exceso de colesterol se acumule en la íntima arterial o revirtiendo una acumulación ya presente. La asociación de bajos niveles séricos de colesterol HDL con un incremento en la incidencia de eventos cardiovasculares isquémicos de origen ateromatoso refleja la importancia del papel protector de esta fracción lipoproteica para la salud cardiovascular. Estudios recientes han demostrado que las partículas de HDL no sólo participan del transporte reverso de colesterol sino que además ejercen un efecto protector sobre el organismo a través de múltiples capacidades que involucran a diferentes componentes lipídicos o proteicos de las HDL o bien que se encuentran asociados a estas lipoproteínas en el plasma.

\section{Capacidad anti-oxidante de las $H D L$}

El estrés oxidativo es una importante condición de riesgo en el desarrollo de enfermedad cardiovascular ya que induce la formación de LDL oxidadas pro-aterogénicas. Diferentes tipos de estudios demuestran que las partículas de HDL circulantes proveen una potente protección contra el daño oxidativo producido por los radicales libres presentes en las LDL, inhibiendo la generación y/o facilitando la degradación de lípidos oxidados proinflamatorios.

Entre otros mecanismos, esta capacidad anti-oxidante de las partículas de HDL se debe a la presencia de enzimas como la PON1 que inhibe la modificación oxidativa de las partículas de $\mathrm{LDL}^{1}$. PON1 cataliza la degradación de los ácidos grasos oxidados presentes en los lípidos de las LDL, evitando así una respuesta inflamatoria caracterizada por producción de citoquinas y adhesión de monocitos a la superficie endotelial. Además, esta enzima reduciría directamente la peroxidación lipídica dentro de las placas ateromatosas coronarias y carotídeas. En modelos animales, se ha demostrado una estrecha relación entre el déficit de PON1 y el desarrollo acelerado de ateroesclerosis. Asimismo, la actividad de PON1 asociada a las HDL se encuentra reducida en enfermedades con elevado estrés oxidativo, como la enfermedad coronaria, las dislipidemias, diversos procesos inflamatorios, diabetes mellitus y ciertas neuropatías ${ }^{2}$.

\section{Capacidad anti-plaquetaria de las HDL}

La formación de un trombo como consecuencia de una placa ateromatosa inestable sería el evento responsable del inicio de la gran mayoría de los síndromes coronarios agudos. El fenómeno trombótico está determinado por distintos elementos (factor tisular, micropartículas apoptóticas, monocitos circulantes) que modulan la trombogenicidad de la placa y la sangre. Asimismo, la activación de las plaquetas y de la cascada de la coagulación está influenciada por las partículas de HDL. Las HDL inhiben la producción de factor tisular, evitando la generación de material pro-coagulante e impiden que el fibrinógeno se una a las plaquetas. Además, se sabe que la formación de trombina se inhibe dramáticamente en presencia de las HDL.

La interacción de las plaquetas -durante su formación o directamente dentro de la circulación- con diferentes lipoproteínas nativas podría determinar un intercambio de lípidos (colesterol y fosfolípidos) que darían cuenta de los cambios observados en la reactividad plaquetaria ${ }^{3}$. Así, datos in vitro han revelado que las LDL son capaces de activar las plaquetas, mientras que las HDL las desensibilizan y ejercen un efecto anti-aterogénico ${ }^{4}$. En este sentido, se ha demostrado que la fracción de HDL3, que constituye la mayor parte de las HDL circulantes, inhibe a la PKC (protein kinase $C$ ) dependiente de fosfatidil-inositol, provocando una disminución de la agregación plaquetaria, de la secreción de gránulos y de la unión a fibrinógeno en respuesta a trombina. El mecanismo que produce este efecto anti-trombótico estaría mediado por el receptor de HDL SR-BI ${ }^{5}$, que se expresa en las plaquetas humanas.

\section{Capacidad anti-apoptótica de las $\mathrm{HDL}$}

El fenómeno de apoptosis consiste en una muerte celular programada que forma parte de diversas condi- 
ciones patológicas, incluyendo la ateroesclerosis. Se ha demostrado que las partículas de HDL inhiben el proceso apoptótico en las células endoteliales, mediante el bloqueo de la vía mitocondrial de la apoptosis. El tratamiento de las células endoteliales con HDL previene la disipación del potencial de la membrana mitocondrial, la liberación de especies reactivas de oxígeno, la liberación de citocromo C hacia el citoplasma y la activación de las caspasas 9 y $3^{6}$. El mecanismo protector de las HDL estaría mediado por la activación de la proteína quinasa Akt, la cual produce señales anti-apoptóticas relacionadas con la inactivación de la vía de la apoptosis mitocondrial ${ }^{6}$. Los componentes de las HDL que activan esta vía de señalización serían la S1P y la apo A-I, que se unen a sus receptores S1P2 y SR-BI, respectivamente ${ }^{7,8}$.

\section{Capacidad anti-inflamatoria de las $\mathrm{HDL}$}

La LDL oxidada y sus componentes modificados inducen la síntesis de citoquinas, mediadores inflamatorios y factores de crecimiento que pueden alterar el comportamiento de las células endoteliales. Una de las primeras manifestaciones de la disfunción endotelial es la expresión de moléculas de adhesión celular (ICAM y VCAM) que promueven el reclutamiento de leucocitos hacia la pared vascular. Los macrófagos y los linfocitos $\mathrm{T}$ localizados en la íntima del vaso inician el proceso inflamatorio que eventualmente se convierte en un proceso nocivo contribuyendo a la progresión de la arteriosclerosis. El efecto protector de las HDL sobre el endotelio ha sido demostrado, tanto mediante el uso de HDL nativa como de HDL reconstituida (rHDL). Como ejemplo, la exposición de las células endoteliales a rHDL hace estas células resistentes a la síntesis de moléculas de adhesión inducida por citoquinas ${ }^{9}$.

\section{Capacidad de transporte de siRNA de las HDL}

Los ácidos ribonucleicos (ARN) de interferencia están siendo reconocidos como un potencial y efectivo método terapéutico debido a la capacidad de estas partículas de silenciar ciertos genes involucrados en diversas patologías. Estudios recientes han demostrado que nanopartículas de HDL son capaces de transportar eficientemente siRNA in vivo y entregarlos a las células mediante la actividad del receptor de HDL SR-BI ${ }^{10}$.

\section{Mecanismos protectores de las HDL frente a un estado séptico}

Las diferentes capacidades de las HDL participan en proteger al organismo de diferentes condiciones patológicas, incluyendo estados sépticos donde las HDL actúan como anti-inflamatorios. Uno de los mecanismos involucrados en esta función ocurriría como resultado de su capacidad de unir lipopolisacárido (LPS) ${ }^{11}$ y facilitar su clearance. Este proceso tendría como consecuencia evitar que se gatille la respuesta inflamatoria mediada por la activación de los receptores del tipo toll (toll like receptors, TLRs) ${ }^{12}$. Estos receptores, presentes en macrófagos, neutrófilos y células dendríticas, reconocen patrones moleculares asociados a patógenos e inician la respuesta inmune innata en el hospedero. Por esto, se ha propuesto que el mecanismo de protección principal de las HDL frente a la acción fisiopatológica del LPS en sepsis, sería la unión y neutralización de éste mediante la inducción de su catabolismo y excreción hepatobiliar ${ }^{13}$.

Específicamente, diversos estudios han mostrado que el LPS adicionado in vitro o liberado in vivo en sepsis por bacterias gramnegativas se podría unir a los distintos tipos de lipoproteínas, incluyendo HDL, LDL y VLDL, existiendo una correlación directa con el contenido fosfolipídico en cada clase lipoproteica ${ }^{14}$. De esta manera, cuando la enzima PLTP (phospholipid transfer protein) une LPS se lo traspasa a las HDL, modificando así la carga fosfolipídica de esta lipoproteína. Por otra parte la enzima CETP al facilitar la transferencia de triglicéridos, colesterol y fosfolípidos entre las distintas lipoproteínas, indirectamente produce que las HDL unan LPS. Adicionalmente, la LBP (LPS binding protein), proteína que media la captación celular del LPS circulante, facilita la presentación de esta partícula a los monocitos para activar la respuesta inmune ${ }^{15}$. De hecho, la mayor parte de LBP circulante se asocia a las HDL ${ }^{16}$. Así, la HDL capta el LPS unido a la LBP atenuando su efecto tóxico, facilitando la entrega de LPS hacia lipoproteínas ricas en triglicéridos, lo que finalmente se traduce en un clearence hepático preferencial del LPS, atenuando la síntesis y liberación de IL-1 $\beta$, IL- 6 y FNT- $\alpha$ estimuladas por LPS desde el sistema monocito-macrófago ${ }^{17}$ (Figura 1).

Otro efecto protector de las HDL en estados sépticos involucra la inhibición de la expresión de moléculas de adhesión e inflamatorias, la reducción del estrés oxidativo y la estimulación de la producción endotelial de óxido nítrico. Las enzimas anti-inflamatorias presentes en las HDL, como la PON1 y PAF-AH, protegerían contra el estrés oxidativo inducido por el estado séptico mediante la producción de mediadores anti-inflamatorios ${ }^{18}$. Esta respuesta se traduciría en la disminución del daño endotelial reflejado en una inhibición de la expresión de moléculas de adhesión junto con una reducción en la liberación de citoquinas pro-inflamatorias ${ }^{19}$. Se ha observado que los niveles de proteína $\mathrm{C}$ reactiva (PCR) -mediador temprano de inflamación- se correlacionan de forma negativa con los niveles de la PON1 ${ }^{18}$, por lo que esta enzima podría ser utilizada como un marcador pronóstico que indique una mayor probabilidad de recuperación de los pacientes sépticos. Consistente con este trabajo, otro estudio demostró que bajos niveles de PON1 se asocian con resultados letales tanto en modelo murino de sepsis como 
en los pacientes sépticos ${ }^{20}$. Adicionalmente, el efecto anti-inflamatorio mediado por las HDL sería potenciado por el hecho de que esta clase de lipoproteínas estimula la producción de óxido nítrico (NO) por activación de la óxido nítrico sintasa endotelial eNOS (endothelial Nitric Oxide Synthase ${ }^{21}$, vía que se encuentra afectada negativamente durante un estado séptico. Sin embargo, existe escasa información en relación al efecto de las HDL sobre la óxido nítrico sintasa inducible iNOS (inducible Nitric Oxide Synthase), la cual tiene alta expresión y actividad en estados sépticos y sería responsable de la hipotensión arterial e hiporeactividad vascular asociada a estos cuadros ${ }^{21}$. Más recientemente, se ha propuesto el posible rol anti-inflamatorio de la apolipoproteína $\mathrm{M}$ (apo $\mathrm{M})$, otro componente proteico funcional de las HDL. De hecho, se observó que a medida que aumenta la gravedad clínica de pacientes con sepsis existe una disminución en los niveles de apo $\mathrm{M}^{22}$. Se ha planteado que el mecanismo que explicaría la relación entre apo M y sepsis dependería de la capacidad de esta apolipoproteína para mediar el transporte de $\mathrm{S}_{1} \mathrm{P}^{22}$, conocido activador de la eNOS y componente de la HDL.

\section{Alteración en los niveles, la composición y la función de HDL inducidos por sepsis}

Es ampliamente conocido que el metabolismo de las lipopoteínas es afectado por las condiciones inflamatorias, incluyendo procesos infecciosos y sépticos. Estos cambios no sólo ocurren en lípidos y apolipoproteínas, sino también en enzimas involucradas en el metabolismo lipoproteico. Las alteraciones en el perfil de lípidos son parte de la respuesta de fase aguda del organismo y, en el caso de los procesos infecciosos, corresponden a la denominada lipemia de la sepsis. Desde un punto de vista lipídico, la sepsis en ratones se asocia a un aumento en los niveles séricos de los triglicéridos, el colesterol total y el colesterol LDL, junto con un descenso en el colesterol HDL. Por otra parte, los pacientes con sepsis presentan un aumento de los niveles de VLDL, pero con un descenso global en el colesterol transportado en LDL y HDL, debido principalmente a una reducción del contenido de ésteres de colesterol de las lipoproteínas. Además, se ha establecido una correlación significativa entre la disminución de los niveles de colesterol HDL y un aumento en la gravedad de la enfermedad séptica, con mayor probabilidad de muerte por sepsis ${ }^{23}$.

El descenso observado, tanto en los niveles de colesterol HDL como en las partículas de HDL en la sepsis, ha sido ampliamente documentado, tanto en modelos animales como en estudios clínicos ${ }^{14}$. Como posibles mecanismos, estudios in vitro han demostrado una reducción en la síntesis de apo A-I en células HepG2 incubadas con citoquinas $^{24}$. Sin embargo, alteraciones en la composición de los lípidos, las enzimas y las apolipoproteínas se perfilan

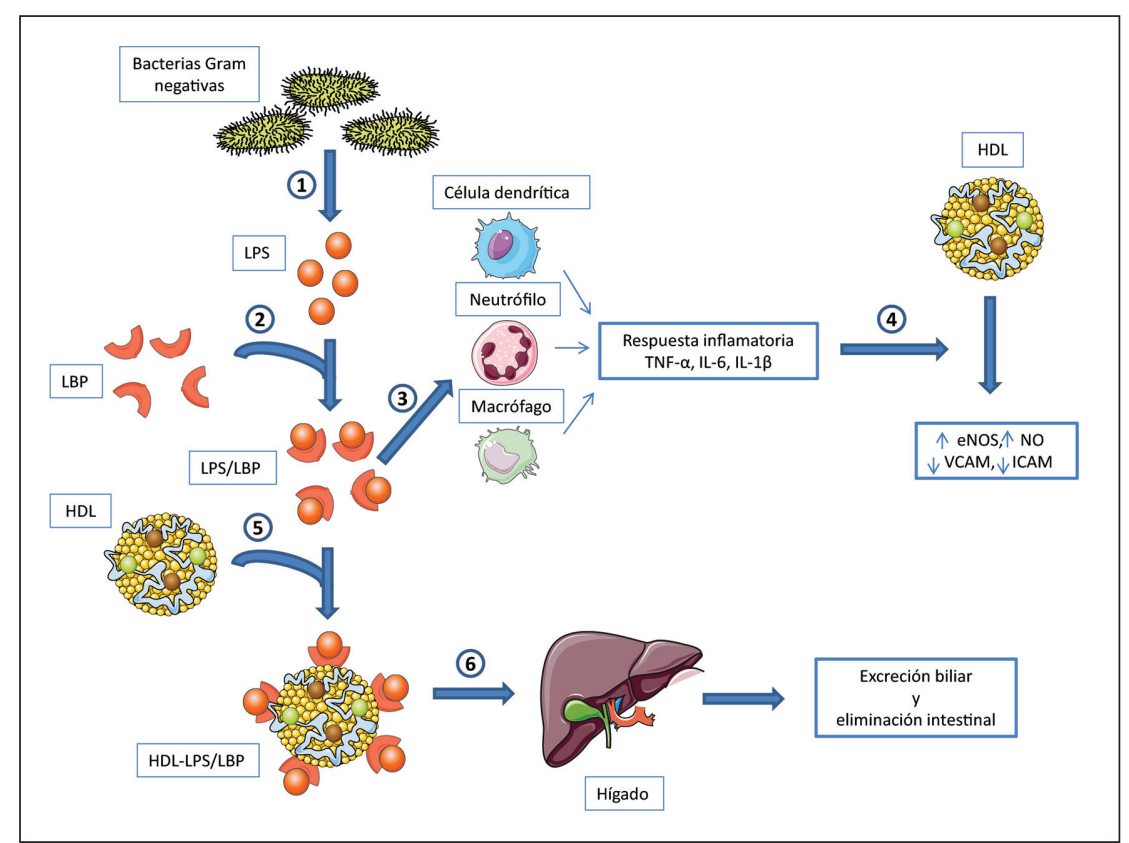

Figura 1.-Efectos protectores de la HDL frente a estados infecciosos y sepsis. Durante una infección por bacterias gramnegativas, se produce la liberación de lipopolisacárido (LPS) hacia el torrente sanguíneo (1), el cual se une a la proteínas ligante de LPS (LBP) (2) y se forma un complejo LPS/LBP. A su vez, este complejo se une a los receptores de tipo Toll (TLR) presentes en células dendríticas, neutrófilos y macrófagos (3) produciendo una activación de la respuesta inmune innata con liberación de factores inflamatorios como TNF- $\alpha$, IL-6 e IL-1 $\beta$. Esta respuesta inflamatoria que genera disfunción endotelial durante un estado séptico se inhibiría mediante la acción de las HDL (4), lo que permitiría una activación de la óxido nítrico (NO) sintasa endotelial (eNOS) y, por lo tanto, en una mayor liberación de NO y una menor expresión y liberación de moléculas de adhesión como VCAM e ICAM. Por otro lado, las partículas de HDL unen el complejo LPS/LBP circulante y canalizan su clearence hepático (6) y posterior eliminación terminal mediante la excreción biliar e intestinal.

como las principales causas que explican la disminución de la protección dada por las HDL durante la sepsis. En efecto, se ha demostrado que las partículas de HDL aumentan el contenido de colesterol no esterificado versus los ésteres de colesterol, se enriquecen en triglicéridos y se observa una disminución de los fosfolípidos totales con un aumento relativo de esfingolípidos ${ }^{14,21}$. Asimismo, las proteínas y apopoliproteínas de las HDL también experimentan modificaciones. La apo A-I es reemplazada por el amiloide sérico tipo A ${ }^{21}$, los niveles de Apo C-I disminuyen, mientras que aumentan los niveles de Apo $\mathrm{E}^{25}$ y fosfolipasa A2 secretora. Estos cambios en la composición de lípidos y apolipoproteínas aceleran el catabolismo terminal de las $\mathrm{HDL}^{26}$. Incluso, estas partículas de HDL presentes durante la sepsis exhiben una mayor afinidad hacia los macrófagos, lo que aparentemente facilitaría la formación de células espumosas y el daño ateromatoso ${ }^{27}$.

En relación a las enzimas asociadas a las HDL, la actividad de LCAT, CETP, PON1 y la HL (hepatic lipase) disminuyen, mientras que la actividad de PLTP aumenta ${ }^{25}$. La resultante de estas alteraciones afecta el metabolismo y funcionalidad de las HDL, generando partículas con un menor contenido de apo A-I, pobres en ésteres de 
colesterol y fosfolípidos y ricas en triglicéridos y en colesterol libre. Además, la disminución de los niveles de LCAT deteriora el eflujo de colesterol desde las células de los tejidos periféricos hacia las HDL, comprometiendo su capacidad de transporte reverso de colesterol. Por otro lado, la menor actividad de CETP disminuye la vía indirecta de este transporte reverso. Ambos cambios impactan desfavorablemente sobre el principal mecanismo anti-aterogénico de las HDL ${ }^{25}$. Adicionalmente, el lipopolisacárido (LPS) disminuye la expresión hepática del receptor de SR-BI en animales y en macrófagos, así como la expresión de ABCA1, ambas proteínas esenciales en el flujo reverso de colesterol desde los tejidos periféricos para su excreción a través del hígado.

Otro aspecto a considerar, son las proteínas con propiedades anti-oxidantes presentes en la HDL. En la respuesta de fase aguda y sepsis, las HDL presentan menor actividad de la enzima PON1 ${ }^{28}$ y de transferrina, proteínas que estarían involucradas en vez de proteína que estaría involucrada en prevenir la oxidación de las partículas LDL ${ }^{27}$. La enzima PAF-AH junto con la PON1, es responsable de prevenir la interacción de los leucocitos con el endotelio mediante la inhibición de la expresión de VCAM y además protege de modificaciones oxidativas a las partículas LDL. Por otra parte, la ceruloplasmina es la principal proteína que une cobre en la sangre y es clásicamente conocida por su función anti-oxidante. A pesar de estar presente en las HDL y exhibir un aumento en su expresión en respuestas de fase aguda, se ha observado que induciría una mayor oxidación en las $\mathrm{LDL}^{27}$ e incluso se ha descrito como marcador de riesgo cardiovascular ${ }^{29}$. De esta manera, el cambio en el perfil anti-oxidante de las HDL como resultado de una desregulación en sus principales enzimas anti-oxidantes, convierte a las HDL en partículas pro-oxidantes y por ende también pro-aterogénicas, lo que impacta negativamente sobre las funciones normales de las HDL como el transporte reverso de colesterol.

\section{Implicancias clínicas de la relación entre HDL y sepsis}

\section{Hipocolesterolemia HDL y bajos niveles de apo A-I como marcador pronóstico en sepsis}

Tradicionalmente, los niveles de colesterol plasmático se han correlacionado con múltiples condiciones de morbi-mortalidad. Epidemiológicamente, mientras la hipercolesterolemia se ha asociado a enfermedades cardiovasculares, la hipocolesterolemia se ha vinculado principalmente a una mayor incidencia de cáncer, infecciones e incluso mortalidad en adultos mayores ${ }^{30}$. Estudios de grupos multiétnicos han descrito una fuerte relación entre hipocolesterolemia y el aumento en distintos tipos de estados infecciosos, incluyendo infecciones genito- urinarias, venéreas, influenza, infecciones respiratorias bajas y bacteriemias ${ }^{30}$.

La investigación clínica más reciente se ha centrado en el análisis de los cambios en el metabolismo y composición de los lípidos y lipoproteínas plasmáticas y su impacto en pacientes críticos, especialmente de origen séptico. La evidencia clínica indica que la mayoría de los pacientes críticos exhiben bajos niveles de colesterol plasmático total ${ }^{31}$. Un estudio descriptivo en una unidad de pacientes críticos quirúrgica analizó los niveles de lípidos al ingreso y los relacionó con los niveles de citoquinas y la evolución posterior. En este estudio, los niveles de colesterol plasmático total fueron inversamente proporcionales a los niveles de IL-6 e IL-10, mientras que los niveles de triglicéridos se correlacionan directamente con los niveles de FNT- $\alpha$. Asimismo, los pacientes que cursaron con hipocolesterolemia presentaron mayor compromiso sistémico, mayor tiempo de estadía en la unidad de cuidado intensivo, mayor susceptibilidad a infecciones post-estabilización y mayor mortalidad. En esta línea, otros estudios han correlacionado directamente la hipocolesterolemia con los niveles de PCR y el índice de mortalidad, tanto en pacientes críticos sépticos y no sépticos, sugiriendo que los niveles séricos de colesterol podrían ser otro marcador de riesgo para identificar pacientes que requieran un tratamiento más agresivo desde el ingreso ${ }^{31}$.

Además del colesterol sanguíneo total, las distintas fracciones de lipoproteínas han sido analizadas en relación a cuadros sépticos. Un estudio retrospectivo analizó la población admitida a un hospital según su nivel de colesterol HDL dividido en dos grupos: colesterol HDL $<20$ o $>65 \mathrm{mg} / \mathrm{dL}$. Así, se estableció que un nivel muy bajo de colesterol HDL se asocia a un mayor riesgo de sepsis y mortalidad intra-hospitalaria, estableciendo que por cada $1 \mathrm{mg} / \mathrm{dL}$ de aumento de colesterol HDL se reducía la mortalidad en $4 \%$ y la sepsis en $11 \%{ }^{32}$. Otro estudio de cohorte y caso-control evaluó a la población admitida en un hospital universitario que evolucionó con sepsis grave comparado con un grupo control. Se demostró que el grupo que evolucionó con sepsis grave presentaba niveles más bajos de colesterol HDL al ingreso en comparación con el grupo control, calculándose que por cada $1 \mathrm{mg} / \mathrm{dL}$ de aumento en el colesterol HDL se asocia una disminución de $3 \%$ en el riesgo de sepsis grave. En este estudio, no se encontró asociación significativa entre niveles de colesterol HDL y riesgo de mortalidad en el grupo séptico, aunque se observó una mayor reducción en los niveles de CETP en los no sobrevivientes comparado con los que se recuperaron de la sepsis ${ }^{33}$.

Además del colesterol HDL, los niveles de apo A-I también han sido estudiados en relación a la sepsis. Un estudio prospectivo observacional en pacientes con criterios de sepsis grave, admitidos a una unidad de pacientes críticos, mostró que los niveles bajos de colesterol HDL y 
de apo A-I al ingreso se correlacionaron con mayor estadía en la unidad, mayor mortalidad y aumento de infecciones intrahospitalarias en los sobrevivientes. Además, tras utilizar un análisis multivariado, los niveles de colesterol HDL $<20 \mathrm{mg} / \mathrm{dL}$ definen un criterio predictor independiente de mortalidad a 30 días, incluso superando el valor predictor de la evaluación APACHE (Acute Physiology and Chronic Health Evaluation) ${ }^{34}$. Otro estudio de similares características indicó que el grupo que falleció por sepsis presentó al ingreso niveles de colesterol total, colesterol HDL, colesterol LDL, apo B, apo A-I y apo C-I más bajos que el grupo sobreviviente. Sin embargo, algunas de estas alteraciones fueron parcialmente revertidas durante la estadía, aunque solamente los niveles de colesterol HDL y los niveles de apo A-I y apo C-I se mantuvieron bajos en el grupo de pacientes fallecidos, estableciendo por regresión logística, que los niveles de apo A-I serían un buen marcador de mortalidad a 30 días asociada a sepsis.

En esta misma línea, un estudio en pacientes cirróticos con sepsis grave observó que los casos que fallecían poseían bajos niveles de colesterol HDL y apo A-I aún después de un ajuste según funcionalidad hepática ${ }^{35}$. Además, este estudio mostró la asociación entre la presencia de bajos niveles de colesterol HDL y apo A-I con altos niveles de IL-6 y FNT- $\alpha$, los cuales se asocian a una enfermedad más grave y caracterizada por mayor compromiso multiorgánico $^{35}$. Importantemente, se estableció que bajos niveles de apo A-I constituyen un marcador independiente de mortalidad a 90 días con un riesgo relativo que supera los scores clínicos SOFA (sequential organ failure assessment) y MELD (model for end-stage liver disease) que indican la gravedad de la enfermedad hepática crónica. De esta forma, los niveles de colesterol HDL y de apo A-I se correlacionan con la reserva hepática y podrían ser usados como nuevos indicadores pronósticos en pacientes cirróticos ante eventuales complicaciones sépticas ${ }^{35}$.

Adicionalmente, un estudio observacional retrospectivo en una unidad de pacientes críticos pediátricos reportó que los pacientes con meningococcemia o shock séptico por $N$. meningitidis presentaron niveles de colesterol total, colesterol LDL y colesterol HDL disminuidos al ingreso, destacando una mayor hipocolesterolemia en pacientes con shock séptico que finalmente fallecieron. Importantemente, los niveles de colesterol HDL se correlacionaron inversamente con los scores SOFA y PRISM (pediatric risk of mortality) y los niveles de IL-6 y FNT- $\alpha$, destacando adicionalmente una asociación entre hipocolesterolemia e hipocortisolismo ${ }^{36}$.

\section{Efecto de HDL recombinante y péptidos miméticos de apo $A-I$ en sepsis}

A pesar que en los últimos años se ha propuesto un potencial uso terapéutico de la administración parenteral de HDL en la enfermedad cardiovascular ateroesclerótica, sus implicancias terapéuticas en sepsis aún no están claramente definidas. Numerosos estudios in vitro, en modelos animales de inflamación y en pacientes con endotoxemia inducida han analizado el potencial rol anti-inflamatorio de las HDL.

Como antecedente, existe evidencia de los efectos de la sobre-expresión de apoA-I. En modelos in vivo, Levine y cols. ${ }^{37}$ realizaron experimentos en ratones manipulados genéticamente para apo A-I humana, los que presentaban concentraciones mayores de colesterol HDL comparado con controles. Al inyectar LPS, los ratones transgénicos con HDL elevadas mostraron una respuesta séptica más retardada, menor liberación de FNT- $\alpha$ y una sobrevida de 3-4 veces mayor que los ratones con menores niveles de HDL. En esta línea, Li y cols. ${ }^{38}$, han reportado que la sobre-expresión de apo A-I en ratón disminuye la inflamación sistémica y daño orgánico múltiple producido por LPS. Este efecto también se correlacionó con una reducción de los niveles de FNT- $\alpha$, IL-6 e IL-1 $\beta$. Por otro lado, recientemente se evaluó la respuesta ante la infección inducida por ligadura y punción cecal en tres grupos de animales: ratones con expresión nula de apo A-I, ratones con sobre-expresión de apo A-I y ratones control que presentaban niveles normales de esta apolipoproteína. A partir de estos experimentos, se observó que la sobrevida y la capacidad de neutralización del LPS estaban disminuidas, mientras que la inducción de citoquinas pro-inflamatorias fue mayor en el grupo que no presentaba la proteína apoA-I con respecto del grupo control. En contraste, se detectó una tendencia a mayor sobrevida en el grupo con sobre-expresión de apo A-I aunque ésta no fue estadísticamente significativa ${ }^{39}$. Este trabajo sugiere que la proteína apo A-I sería importante en contrarrestar la respuesta inflamatoria asociada a este modelo específico de sepsis. De esta forma, los modelos de endotoxemia y de sepsis muestran la importancia de apoA-I y de altos niveles de HDL en el clearence de LPS, la inflamación, el riesgo de daño multiorgánico y la sobrevida.

Así como la expresión transgénica de apo A-I muestra una disminución de la respuesta sistémica ante LPS, múltiples estudios han sido reportados con resultados similares con el uso de rHDL. Estudios in vitro han evaluado los efectos de rHDL sobre muestras de sangre de pacientes críticos y sujetos sanos, ambas incubadas con LPS. En ambos grupos, la incubación conjunta de LPS con rHDL y de rHDL indujo una supresión de la respuesta inflamatoria inducida por LPS y medida por los niveles de FNT- $\alpha$, sugiriendo que rHDL fue capaz de neutralizar la respuesta endotóxica in vitro. Por su parte, en modelos animales de endotoxemia, se ha establecido que el aumento en los niveles de FNT- $\alpha$ inducido por LPS es atenuado mediante el pre-tratamiento con rHDL, aumentando la sobrevida de los animales ${ }^{37}$. Por otra parte, en un modelo de bacteriemia inducida por especies gramnegativas, los niveles de 
FNT- $\alpha$ disminuyen ante un pre-tratamiento con rHDL lo que se asocia con efectos hemodinámicos positivos. Sin embargo, estos efectos anti-inflamatorios no se observan cuando el tratamiento con rHDL ocurre con posterioridad a la inducción de la bacteriemia, aunque se mantienen los efectos hemodinámicos favorables ${ }^{40}$.

Adicionalmente, se han evaluado los efectos en daño orgánico múltiple con el uso de rHDL en modelos animales de endotoxemia. En estos estudios se ha observado que el pre-tratamiento con rHDL reduce el daño estructural renal, hepático, pulmonar e intestinal producido por $\mathrm{LPS}^{41}$. Aunque en este estudio no se detectó una disminución de la elevación de los niveles de FNT- $\alpha$ inducidos por LPS, la rHDL disminuyó la expresión glomerular renal de P-selectina e ICAM, sugiriendo que en este modelo el mecanismo protector de las rHDL sería mediante la inhibición de las moléculas de adhesión presentes en la superficie celular. Consecuentemente, estudios in vitro han reportado que el uso de rHDL en cultivos de células endoteliales induce una menor expresión de las moléculas de adhesión VCAM-1 e ICAM-1 en respuesta a la exposición de estas células a FNT- $\alpha$ o LPS ${ }^{42}$.

Contrariamente a lo observado en modelos de endotoxemia, un modelo de perros con shock séptico secundario a peritonitis por Escherichia coli mostró que el uso de rHDL post-inoculación bacteriana disminuye efectivamente los niveles plasmáticos de LPS, FNT- $\alpha$ y la leucopenia inducida post-infección. Sin embargo, se asoció a efectos adversos como alza en transaminasas hepáticas, convulsiones y no mostró efectos en la sobrevida animal ${ }^{43}$. Este estudio es importante puesto que no se observaron efectos positivos sobre la mortalidad, pero sí otros efectos adversos, lo que es interesante de analizar ya que denota la diferencia entre los modelos de endotoxemia y sepsis, así como la distinción entre el uso de rHDL antes o después de instaurada la sepsis.

En humanos, varios estudios han abordado los efectos del uso profiláctico de rHDL en pacientes con endotoxemia inducida. Pajkrt y cols., describieron que el uso de rHDL induce una disminución en la sintomatología asociada a un cuadro febril, además de una menor alza en los niveles de FNT- $\alpha$, IL-6, IL-8 y una menor leucocitosis. Adicionalmente, el uso de rHDL induce una reducción en la expresión de CD14 en monocitos, el principal receptor de LPS en estas células ${ }^{44}$. En otro estudio, se evaluó la prevención mediada por el uso de rHDL sobre el efecto pro-coagulante causado por el tratamiento con LPS en humanos ${ }^{45}$. Efectivamente, las rHDL, además de disminuir la activación de protrombina, redujeron los niveles del activador del plasminógeno tisular y la agregación plaquetaria inducida por colágeno, lo cual implicaría un mejor control de los efectos pro-coagulantes asociados a los cuadros de sepsis ${ }^{45}$.

Por otro lado, Levels y cols., determinaron los cambios en el metabolismo lipídico por el uso profiláctico de rHDL en sujetos sanos expuestos a endotoxina. Además de observar efectos similares en cuanto a una reducción de sintomatología, disminución de citoquinas y factores pro-coagulantes tal como en estudios previos, analizaron los cambios en la composición de lípidos y proteínas que podrían explicar estos efectos. En el grupo pre-tratado con rHDL, se observó un aumento de los niveles de colesterol total, apo A-I y colesterol HDL. Sin embargo, las rHDL no previnieron la caída esperada en los lípidos tras la infusión de LPS, pero se indujo un aumento en el contenido de fosfolípidos en las fracciones de VLDL, LDL y HDL y un aumento en la actividad de la enzima PLTP, sugiriendo una mayor unión de LPS a estas partículas y con ello un mayor clearence hepático de la endotoxina.

En humanos no existen estudios que reporten efectos adversos del uso de rHDL en modelos de endotoxemia. No obstante, se han descrito discretas elevaciones de transaminasas sin repercusión clínica en estudios clínicos de protección cardiovascular. En este sentido, un estudio clínico observó que pacientes expuestos a altas dosis de rHDL, pero no a dosis moderadas o bajas, presentaban un aumento del crecimiento de Candida albicans en el plasma, sugiriendo un uso cuidadoso de rHDL en cuadros de septicemia con agente causal desconocido ${ }^{46}$.

Al igual que rHDL, los péptidos miméticos de apo A-I parecen ejercer protección cardiovascular anti-aterogénica en modelos animales. Sin embargo, su uso en modelos de endotoxemia y sepsis ha sido más limitado. Estos péptidos son secuencias aminoacídicas correspondientes a estructuras de las $\alpha$-hélices anfipáticas presentes en la apo A-I con alta afinidad por lípidos, en especial, por ácidos grasos oxidados pro-inflamatorios ${ }^{28}$. En este sentido, se ha demostrado que el uso del péptido mimético L-4F de apo A-I revierte el aumento de los niveles de las citoquinas pro-inflamatorias IL-8, IL-6, FNT- $\alpha$ e IFN- $\gamma$ inducido por LPS en células endoteliales y en ratas ${ }^{47}$. Además, este péptido reduce la adhesión de monocitos a las células endoteliales inducida por LPS y mediada por VCAM. Se ha propuesto que los mecanismos por los cuales L-4F protegería contra los efectos de LPS serían su unión directa y neutralización, induciendo aumento en los niveles de HDL o bien facilitando su captación por las partículas de $\mathrm{HDL}^{48}$. En esta misma línea, utilizando un modelo de sepsis con ligadura y punción cecal se demostró que el péptido L-4F disminuyó el aumento de los niveles plasmáticos de IL-6 inducidos por esta condición experimental. Importantemente, la disminución en los niveles de colesterol HDL inducidos en este modelo fue atenuada por la acción del péptido mimético. Recientemente, Dai y cols., evaluaron los efectos de L-4F sobre la reactividad vascular en un modelo de endotoxemia. El uso terapéutico del péptido mimético disminuyó la hipotensión arterial observada tras la administración de LPS, indujo una menor actividad de 
iNOS y una disminución en los niveles de metabolitos del óxido nítrico, con una menor mortalidad en el grupo tratado. En este mismo estudio, el análisis ex vivo de reactividad vascular en aortas mostró que $\mathrm{L}-4 \mathrm{~F}$ revierte la disminución de la actividad contráctil mediada por fenilefrina observada en el grupo con LPS. Esto sugeriría que el efecto de L-4F, sería a través de la mejoría de la hiporeactividad vascular inducida por iNOS. ${ }^{48}$.

En esta línea, otro estudio analizó el efecto del péptido L-4F sobre la función cardíaca en ratas. Al inducir endotoxemia, se observó una caída en la precarga, reflejada en una disminución del volumen ventricular al fin de diástole, con consecuente disminución en el gasto cardiaco. El uso de L-4F revirtió estos efectos y se observó un aumento de volumen ventricular en fin de diástole con mejoría en el gasto cardiaco. Otro estudio evaluó el efecto del péptido L-4F sobre el daño pulmonar agudo en ratas endotoxémicas. Estos autores determinaron que el péptido L-4F atenúa de manera significativa el daño del tejido pulmonar y disminuye la mortalidad de las ratas tratadas con LPS. Asimismo, el péptido revierte de forma significativa el aumento en los niveles de las moléculas de adhesión ICAM y E-selectina y la activación de las vías de señalización

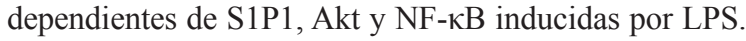
Adicionalmente, observaron que el péptido L-4F produce un aumento del colesterol HDL, lo que probablemente explica el incremento en la sobrevida de los animales.

Finalmente, Sharivof y cols., evaluaron los efectos de L-4F en la sobrevida animal en un modelo de endotoxemia. En este estudio, un grupo de animales recibió de manera conjunta L-4F y LPS, otro grupo animal se trató primero con LPS y una hora post-infusión de LPS se administró L-4F, un tercer grupo fue infundido solamente con LPS y un cuarto grupo correspondió a la condición control. A partir de este experimento se determinó que la sobrevida animal fue mayor si el péptido L-4F era infundido conjuntamente a LPS, aunque igualmente se observó beneficio si el péptido era infundido con posterioridad al LPS, respecto del grupo no expuesto a L-4F. En esta misma publicación, además de corroborar los efectos de L-4F sobre los niveles de citoquinas y de colesterol sanguíneo descritos en otros estudios anteriormente comentados, los autores analizaron el efecto del LPS sobre la actividad anti-oxidante de las HDL mediante la medición de la actividad de la enzima PON1. Como se esperaba, la infusión de LPS disminuyó la actividad de PON1 respecto del grupo control. Sin embargo, el grupo tratado con LPS y luego de una hora de infusión de L-4F mostró que la actividad de PON1 no experimenta cambios significativos en comparación al grupo control y es significativamente mayor a la observada en el grupo tratado sólo con LPS 49. Este estudio es relevante porque muestra que la utilización del péptido L-4F presenta beneficios aun después de instaurada la endotoxemia. Más interesante aún es el hecho que se demuestra un efecto benéfico del uso de este péptido mimético sobre las propiedades anti-oxidantes de las HDL y la sobrevida animal.

Si bien estas formas análogas de HDL presentan resultados prometedores en modelos de endotoxemia a nivel de marcadores inflamatorios y con efectos fisiológicamente favorables, existe poca evidencia publicada sobre su utilidad en relación a modelos de sepsis. Como hemos discutido, la sepsis es un proceso complejo que involucra múltiples mecanismos y cambios asociados, muchos de ellos mediados por LPS, aunque no es el único agente gatillante y patogénico participante. Así, Quezado y cols., usando un modelo canino de shock séptico, demostraron que el uso de rHDL efectivamente disminuye los marcadores de inflamación, pero esto no se correlacionó con una mayor sobrevida en el grupo tratado ${ }^{43}$. En otro estudio, utilizando un modelo de bacteriemia por especies gramnegativas asociado a terapia con $\mathrm{rHDL}^{40}$, reportó solamente estudios fisiológicos, sin analizar resultados como mortalidad o disfunción de órganos. De hecho, existe una sola publicación, basada en un modelo quirúrgico de sepsis con ligadura y punción cecal y uso de L-4F a las $6 \mathrm{~h}$ de realizada la ligadura. En éste estudio, se observó un resultado favorable del uso terapéutico de este péptido mimético de apo A-I sobre la sobrevida a $48 \mathrm{~h}$, siendo este resultado prometedor para implicancias terapéuticas de este análogo.

Como se comentó anteriormente, la capacidad de neutralizar LPS por las lipoproteínas está relacionada con su contenido de fosfolípidos. Además del uso de rHDL y L-4F, las infusiones de lípidos se han estudiado con el objetivo descubrir nuevos agentes terapéuticos en sepsis. Entre estas, el uso de soluciones lipídicas ricas en fosfolípidos muestran efectos favorables como reversión de la lipemia de la sepsis, neutralización de LPS, disminución de citoquinas pro-inflamatorias y mayor sobrevida en modelos animales de endotoxemia y sepsis, así como en modelos de endotoxemia en sujetos sanos, donde se observa disminución en sintomatología asociada a este cuadro ${ }^{50}$.

Dado los resultados prometedores de estas soluciones con fosfolípidos, incluyendo su seguridad en un estudio fase $\mathrm{I}^{50}$, se diseñó y desarrolló el protocolo LIPOS (lipid infusion and patient outcomes in sepsis) $)^{50}$, un estudio clínico randomizado, ciego, contra placebo, multicéntrico, de tres ramas: placebo, dosis bajas y dosis altas de emulsión de fosfolípidos aplicada en infusión continua durante tres días en pacientes con sepsis grave confirmada o sospechada por bacterias gramnegativas con $<36 \mathrm{~h}$ de inicio de antimicrobianos $\mathrm{y}<12 \mathrm{~h}$ desde la aparición de disfunción orgánica. Al año de estudio, la rama que usó dosis alta de la emulsión fue cancelada por efectos adversos serios, mientras que su aplicación en dosis bajas no mostró un impacto favorable sobre la mortalidad ni la incidencia de nuevos eventos de fallas orgánica. La ausencia de efecto podría explicarse porque la neutralización del 
LPS debe aplicarse más precozmente durante el proceso infeccioso o porque la infusión de lípidos no se incorporó efectivamente en las lipoproteínas, las cuales son claves como tales -junto con sus componentes proteicos- en la acción protectora frente a la sepsis.

En resumen, se requieren nuevos estudios pre-clínicos en modelos de sepsis, más allá de endotoxemia inducida por LPS, para evaluar los posibles efectos benéficos de estos análogos de HDL en un sistema más integrado y así en un futuro evaluar la factibilidad de realizar nuevos estudios clínicos con miras a establecer un rol terapéutico de estos agentes en clínica humana.

\section{Conclusiones}

Las lipoproteínas HDL poseen diversas actividades además del transporte reverso del colesterol entre las que se encuentran la actividad anti-oxidante, anti-agregante plaquetaria, anti-apoptótica, anti-inflamatoria, inactivante de lipopolisacárido y transportadora de siRNA. En diferentes condiciones patológicas, incluyendo estados sépticos, las diferentes funciones de las HDL actúan en forma concertada para proteger al organismo. Además, la evidencia indica que los niveles de HDL presentes en fases agudas de un estado séptico se reducen significativamente y que esta caída podría tener un papel patogénico y valor pronóstico en la evolución de este tipo de patología. En los diversos estudios revisados, queda en evidencia que al menos dos de los componentes de las HDL serían los responsables de su efecto anti-séptico: la apo A-I y la enzima PON1. Sin embargo, todavía no se conoce con precisión los mecanismos moleculares y celulares detrás de este efecto anti-inflamatorio inducido por los otros componentes de las HDL. La administración de péptidos miméticos de la apo A-I o rHDL reduce la respuesta in- flamatoria, mejora el pronóstico y sobrevida en diferentes modelos animales de endotoxemia. No obstante, todavía existe poca evidencia que sustente el beneficio del uso de HDL o similares en sepsis humana. Por lo tanto, se requieren estudios clínicos adicionales que validen a las HDL como un nuevo blanco terapéutico para el manejo de los procesos inflamatorios asociados a la sepsis.

\section{Resumen}

Las lipoproteínas de alta densidad (HDL) son responsables del transporte reverso de colesterol y ejercen un importante papel anti-aterogénico. En los últimos años, diversos estudios indican que las HDL también tendrían otras funciones críticas, incluyendo una posible actividad anti-inflamatoria durante estados infecciosos. Además, la evidencia disponible sugiere que la presencia de lipopolisacárido (LPS) en la circulación durante estados infecciosos inducidos por bacterias gramnegativas podría estar involucrado en la disminución del colesterol HDL y los cambios en composición de esta clase lipoproteínas, lo cual se asociaría con una mayor tasa de mortalidad por sepsis en modelos animales y en humanos. En este trabajo, se revisan los antecedentes mencionados y además se discuten posibles mecanismos que explican la disminución de la respuesta inflamatoria y de la mortalidad que se logran en modelos de endotoxemia tratados con HDL o preparaciones similares. En este sentido, se ha propuesto que uno de los mecanismos protectores de las HDL estaría mediado por su capacidad de unión y/o neutralización del LPS, evitando una respuesta exacerbada del sistema inmune. De esta manera, el aumento de los niveles sanguíneos de HDL y/o su administración parenteral podrían constituir nuevas herramientas anti-inflamatorias para el manejo de estados sépticos en humanos.

\section{Referencias bibliográficas}

1.- Fuhrman B, Volkova N, Aviram M. Paraoxonase 1 (PON1) is present in postprandial chylomicrons. Atherosclerosis 2005; 180: 55-61.

2.- Gowri M S, Van der Westhuyzen D R, Bridges $\mathrm{S}$ R, Anderson J W. Decreased protection by HDL from poorly controlled type 2 diabetic subjects against LDL oxidation may be due to the abnormal composition of HDL. Arterioscler Thromb Vasc Biol 1999; 19: 2226-33.

3.- Siegel-Axel D, Daub K, Seizer P, Lindemann S, zGawaz M. Platelet lipoprotein interplay: trigger of foam cell formation and driver of atherosclerosis. Cardiovasc Res 2008; 78: 8-17.

4.- Surya I I, Akkerman J W. The influence of lipoproteins on blood platelets. Am Heart J. 1993; 125: 272-5.

5.- Brodde M F, Korporaal S J, Herminghaus G, Fobker M, Van Berkel T J, Tietge UJ, et al. Native high-density lipoproteins inhibit platelet activation via scavenger receptor BI: role of negatively charged phospholipids. Atherosclerosis 2011; 215: 374-82.

6.- Nofer J R, Levkau B, Wolinska I, Junker R, Fobker M, von Eckardstein A, et al. Suppression of endothelial cell apoptosis by high density lipoproteins (HDL) and HDL-associated lysosphingolipids. J Biol Chem 2001; 14: 34480-5.

7.- $\quad$ Frias M A, Lang U, Gerber-Wicht C, James $\mathrm{R}$ W. Native and reconstituted HDL protect cardiomyocytes from doxorubicin-induced apoptosis. Cardiovasc Res 2010; 85: 118-26.

8.- Fuhrman B, Gantman A, Aviram M. Paraoxonase 1 (PON1) deficiency in mice is associated with reduced expression of macrophage SR-BI and consequently the loss of HDL cytoprotection against apoptosis. Atherosclerosis 2010; 211: 61-8.
9.- Clay M A, Pyle D H, Rye K A, Vadas M A, Gamble J R, Barter P J. Time sequence of the inhibition of endothelial adhesion molecule expression by reconstituted high density lipoproteins. Atherosclerosis 2001; 157: 23-9.

10.- Shahzad M M, Mangala L S, Han H D, Lu C, Bottsford-Miller J, Nishimura M, et al. Targeted delivery of small interfering RNA using reconstituted high-density lipoprotein nanoparticles. Neoplasia 2011; 13: 309-19.

11.- Emancipator K, Csako G, Elin RJ. In vitro inactivation of bacterial endotoxin by human lipoproteins and apolipoproteins. Infect Immun 1992; 60: 596-601.

12.- O’Mahony D S, Pham U, Iyer R, Hawn T R, Liles W C. Differential constitutive and cytokine-modulated expression of human toll-like receptors in primary neutrophils, monocytes, and macrophages. Int J Med Sci 2008; 4: 1-8. 
13.- Thompson P A, Kitchens R L. Native highdensity lipoprotein augments monocyte responses to lipopolysaccharide (LPS) by suppressing the inhibitory activity of LPSbinding protein. J Immunol 2006; 177: 4880-7.

14.- Kitchens R L, Thompson P A, Munford R S, O'Keefe G E. Acute inflammation and infection maintain circulating phospholipid levels and enhance lipopolysaccharide binding to plasma lipoproteins. J Lipid Res 2003; 44: 2339-44.

15.- Kirschning C J, Au-Young J, Lamping N, Reuter D, Pfeil D, Seilhamer J J, et al. Similar organization of the lipopolysaccharide-binding protein (LBP) and phospholipid transfer protein (PLTP) genes suggests a common gene family of lipid-binding proteins. Genomics 1997; 46: 416-25.

16.- Levels J H, Abraham P R, van Barreveld E P, Meijers J C, van Deventer S J. Distribution and kinetics of lipoprotein-bound lipoteichoic acid. Infect Immun 2003; 71: 3280-4.

17.- Cavaillon J M, Fitting C, Haeffner-Cavaillon N, Kirsch S J, Warren H S. Cytokine response by monocytes and macrophages to free and lipoprotein-bound lipopolysaccharide. Infect. Immun 1990; 58: 2375-82.

18.- Novak F, Vavrova L, Kodydkova J, Novak F Sr, Hynkova M, Zak A, et al. Decreased paraoxonase activity in critically ill patients with sepsis. Clin Exp Med 2010; 10: 21-5.

19.- Cockerill G W, Rye K A, Gamble J R, Vadas M A, Barter P J. High density lipoproteins inhibit cytokine-induced expression of endothelial cell adhesion molecules. Arterioscler Thromb Casc Biol 1995; 15: 1987-94.

20.- Draganov D, Teiber J, Watson C, Bisgaier C, Nemzek J, Remick D, et al. PON1 and oxidative stress in human sepsis and an animal model of sepsis. Adv Exp Med Biol 2010; 660: 89-97.

21.- Wu A, Hinds C, Thiemermann C. Highdensity lipoproteins in sepsis and septic shock: Metabolism, actions, and therapeutic applications. Shock 2004; 21: 210-21.

22.- Christoffersen C, Nielsen L B. Apolipoprotein M-a new biomarker in sepsis. Crit Care 2012; 16: 126.

23.- Shor R Wainstein J, Oz D, Boaz M, Matas Z, Fux A, et al. Low HDL levels and the risk of death, sepsis and malignancy. Clin Res Cardiol 2008; 97: 227-33.

24.- Ettinger W H, Varma V K, Sorci-Thomas M, Parks J S, Sigmon R C, Smith T K, et al. Cytokines decrease apolipoprotein accumulation in medium from Hep G2 cells. Arterioscler Thromb 1994; 14: 8-13.

25.- Barlage S, Fröhlich D, Böttcher A, Jauhiainen M, Müller HP, Noetzel F, et al. ApoE-containing high density lipoproteins and phospholipid transfer protein activity increase in patients with a systemic inflammatory response. J Lipid Res 2001; 42: 281-90.

26.- Cabana V G, Siegel J N, Sabesin S M. Effects of the acute phase response on the concentration and density distribution of plasma lipids and apolipoproteins. J Lipid Res 1989; 30: 39-49.

27.- Khovidhunkit W, Memon R, Feingold K, Grunfeld C. Infection and inflammation-induced proatherogenic changes of lipoproteins. J Infect Dis 2000; 181: 462-72.

28.- Van Lenten B J, Navab M, Anantharamaiah G M, Buga G M, Reddy S T, Fogelman A M. Multiple indications for anti-inflammatory apolipoprotein mimetic peptides. Curr Opin Investig Drugs 2008; 9: 1157-62.

29.- Mänttäri M, Manninen V, Huttunen J K, Palosuo T, Ehnholm C, Heinonen O P, et al. Serum ferritin and ceruloplasmin as coronary risk factors. Eur Heart J 1994; 15: 1599-603.

30.- Iribarren C, Jacobs D R Jr, Sidney S, Claxton A J, Gross M D, Sadler M, et al. Serum total cholesterol and risk of hospitalization, and death from respiratory disease. Int J Epidemiol 1997; 26: 1191-202.

31.- Giovannini I, Boldrini G, Chiarla C, Giuliante F, Vellone M, Nuzzo G. Pathophysiologic correlates of hypocholesterolemia in critically ill surgical patients. Intensive Care Med 1999; 25: 748-51.

32.- Shor R, Wainstein J, Oz D, Boaz M, Matas Z, Fux A, et al. Low HDL levels and the risk of death, sepsis and malignancy. Clin Res Cardiol 2008; 97: 227-33.

33.- Grion C M, Cardoso L T, Perazolo T F, García A S, Barbosa D S, Morimoto H K, et al. Lipoproteins and CETP levels as risk factors for severe sepsis in hospitalized patients. Eur J Clin Invest 2010; 40: 330-8.

34.- Chien J Y, Jerng J S, Yu C J, Yang P C. Low serum level of high-density lipoprotein cholesterol is a poor prognostic factor for severe sepsis. Crit Care Med 2005; 33: 1688-93.

35.- Tsai M H, Peng Y S, Chen Y C, Lien J M, Tian Y C, Fang J T, et al. Low serum concentration of apolipoprotein A-I is an indicator of poor prognosis in cirrhotic patients with severe sepsis. J Hepatol 2009; 50: 906-15.

36.- Vermont C L, den Brinker M, Kâkeci N, de Kleijn E D, de Rijke Y B, Joosten K F, et al. Serum lipids and disease severity in children with severe meningococcal sepsis. Crit Care Med 2005; 33: 1610-5.

37.- Levine $\mathrm{D} M$. In vivo protection against endotoxin by plasma high density lipoprotein. Proc Natl Acad Sci USA 1993; 90: 12040-4.

38.- Li Y, Dong J B, Wu M P. Human ApoA-I overexpression diminishes LPS-induced systemic inflammation and multiple organ damage in mice. Eur J Pharmacol 200; 590: 417-22.

39.- Guo L, Ai J, Zheng Z, Howatt D A, Daugherty A, Huang B, et al. High density lipoprotein protects against polymicrobe-induced sepsis in mice. J Biol Chem 2013; 288: 17947-53.

40.- Casas A T, Hubsch A P, Doran J E. Effects of reconstituted high-density lipoprotein in persistent gram-negative bacteremia. Am Surg 1996; 62: 350-5.

41.- McDonald M C, Dhadly P, Cockerill G W, Cuzzocrea S, Mota-Filipe H, Hinds C J, et al. Reconstituted high-density lipoprotein attenuates organ injury and adhesion molecule expression in a rodent model of endotoxic shock. Shock. 2003; 20: 551-7.

42.- Calabresi L, Franceschini G, Sirtori C R, De Palma A, Saresella M, Ferrante P, et al. Inhibition of VCAM-1 expression in endothelial cells by reconstituted high density lipoproteins. Biochem Biophys Res Commun 1997; 238: 61-5.

43.- Quezado Z M, Natanson C, Banks S M, Alling D W, Koev C A, Danner R L, et al. Therapeutic trial of reconstituted human highdensity lipoprotein in a canine model of gramnegative septic shock. J Pharmacol Exp Ther 1995; 272: 604-11.

44.- Pajkrt D, Doran J E, Koster F, Lerch P G, Arnet B, van der Poll T, et al. Antiinflammatory effects of reconstituted high-density lipoprotein during human endotoxemia. J Exp Med 1996; 184: 1601-8.

45.- Pajkrt D, Lerch P G, van der Poll T, Levi M, Illi M, Doran J E, et al. Differential effects of reconstituted high-density lipoprotein on coagulation, fibrinolysis and platelet activation during human endotoxemia. Thromb Haemost 1997; 77: 303-7.

46.- Netea M G, Curfs J H, Demacker P N, Meis J F, Van der Meer J W, Kullberg B J. Infusion of lipoproteins into volunteers enhances the growth of Candida albicans. Clin Infect Dis 1999; 28: 1148-51.

47.- Gupta H, Dai L, Datta G, Garber D W, Grenett H, Li Y, et al. Inhibition of lipopolysaccharide-induced inflammatory responses by an apolipoprotein AI mimetic peptide. Circ Res 2005; 97: 236-43.

48.- Dai L, Datta G, Zhang Z, Gupta H, Patel R, Honavar J, et al. The apolipoprotein A-I mimetic peptide $4 \mathrm{~F}$ prevents effects in vascular function in endotoxemic rats. J Lipid Res 2010; 51: 2695-705.

49.- Sharifov O F, Xu X, Gaggar A, Grizzle W E, Mishra V K, Honovar J, et al. Anti-inflammatory mechanisms of apolipoprotein A-I mimetic peptide in acute respiratory distress syndrome secondary to sepsis. PLoS ONE 2013; 8: e64486.

50.- Dellinger R P, Tomayko J F, Angus D C, Opal S, Cupo M A, McDermontt S, et al. The Lipid Infusion and Patient Outcomes in Sepsis (LIPOS) Investigators. Efficacy and safety a phospholipid emulsion (GR270773) in gramnegative severe sepsis: Results of a phase II multicenter, randomized, placebo-controlled, dose-finding clinical trial. Crit Care Med 2009; 37: 2929-38 